\title{
Selection of Vaccinia Virus-Neutralizing Antibody from a Phage-Display Human-Antibody Library
}

\author{
Yong Won Shin ${ }^{1 *}$, Ki-Hwan Chang ${ }^{1}$, Gwang-Won Hong ${ }^{1}$, Sang-Gu Yeo ${ }^{2}$, Youngmee Jee ${ }^{2}$, Jong-Hyun Kim ${ }^{2,3}$, \\ Myoung-don $\mathrm{Oh}^{4}$, Dong-Hyung $\mathrm{Cho}^{5}$, and Se-Ho Kim ${ }^{6,7 *}$
}

\author{
${ }^{1}$ RED Center, GC Pharma, Yongin 16924, Republic of Korea \\ ${ }^{2}$ Division of VPD Control and NIP, Korea Centers for Disease Control and Prevention, Ministry of Health and Welfare, Osong Health \\ Technology Administration Complex, Cheongju 28159, Republic of Korea \\ ${ }^{3}$ Department of Pediatrics, The Catholic University of Korea College of Medicine, St. Vincent's Hospital, Suwon 16247, Republic of Korea \\ ${ }^{4}$ Department of Internal Medicine, College of Medicine, Seoul National University, Seoul 03080, Republic of Korea \\ ${ }^{5}$ School of Life Science, Kyungpook National University, Daegu 41566, Republic of Korea \\ ${ }^{6}$ Division of Biomedical Convergence, College of Biomedical Science, Kangwon National University, Chuncheon 24341, Republic of Korea \\ ${ }^{7}$ Institute of Bioscience and Biotechnology, Kangwon National University, Chuncheon 24341, Republic of Korea
}

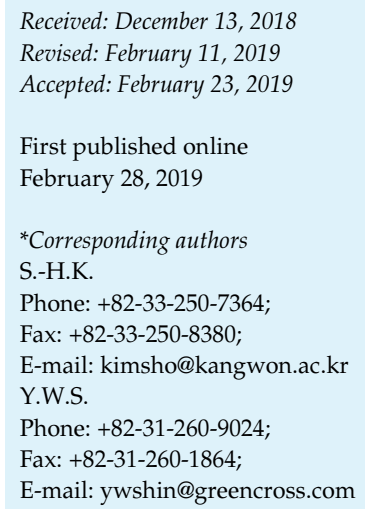

\begin{abstract}
Although smallpox was eradicated in 1980, it is still considered a potential agent of biowarfare and bioterrorism. Smallpox has the potential for high mortality rates along with a major public health impact, eventually causing public panic and social disruption. Passive administration of neutralizing monoclonal antibodies (mAbs) is an effective intervention for various adverse reactions caused by vaccination and the unpredictable nature of emerging and bioterroristrelated infections. Currently, vaccinia immune globulin (VIG) is manufactured from vaccinia vaccine-boosted plasma; however, this production method is not ideal because of its limited availability, low specific activity, and risk of contamination with blood-borne infectious agents. To overcome the limitations of VIG production from human plasma, we isolated two human single-chain variable fragments (scFvs), (SC34 and SC212), bound to vaccinia virus (VACV), from a scFv phage library constructed from the B cells of VACV vaccine-boosted volunteers. The scFvs were converted to human IgG1 (VC34 and VC212). These two antiVACV mAbs were produced in Chinese Hamster Ovary (CHO) DG44 cells. The binding affinities of VC34 and VC212 were estimated by competition ELISA to $\mathrm{IC}_{50}$ values of $2 \mu \mathrm{g} / \mathrm{ml}$ (13.33 $\mathrm{nM})$ and $22 \mu \mathrm{g} / \mathrm{ml}(146.67 \mathrm{nM})$, respectively. Only the VC212 mAb was proven to neutralize the VACV, as evidenced by the plaque reduction neutralization test (PRNT) result with a $\mathrm{PRNT}_{50}$ of $\sim 0.16 \mathrm{mg} / \mathrm{ml}(\sim 1.07 \mu \mathrm{M})$. This VC212 could serve as a valuable starting material for further development of VACV-neutralizing human immunoglobulin for a prophylactic measure against post-vaccination complications and for post-exposure treatment against smallpox.
\end{abstract}

Keywords: Monoclonal antibody, phage display, single-chain variable fragment (scFv), smallpox, vaccinia virus, vaccinia immune globulin

\section{Introduction}

Smallpox is caused by the variola virus (VARV), which belongs to the Orthopoxvirus genus. Vaccinia and cowpox viruses are also included in the same genus as VARV [1]. It is known that cross-protection in the Orthopoxvirus genus is possible, and therefore smallpox can be prevented by vaccinia virus (VACV) vaccination [2].

The World Health Organization (WHO) declared that smallpox was eradicated in 1980 and smallpox vaccination 
was discontinued in the early 1980s. However there exists the possibility of bioterror threat with VARV [3], and smallpox vaccination was reintroduced in response to this risk. Concomitantly, vaccinia immune globulin (VIG) was required for management and possible prophylaxis for vaccine-related serious adverse events [4].

Currently VIG is prepared from plasma with high titers of anti-vaccinia immunoglobulin. The donors for plasma are boosted with a VACV vaccine to raise the titers of antivaccinia immunoglobulin [5]. However, the production of VIG from vaccinated human plasma entails inconvenience in collection. In addition, the availability of high-titer plasma is limited, and there exists the possibility of contamination with infectious agents [6].

VACV-specific human monoclonal antibodies (mAbs) can replace VIG, which can eliminate the risks typically associated with human blood products and provide a wellcharacterized, high-quality immune globulin [6]. Several experimental approaches are currently available for generating human mAbs, and phage display is the most widely used technology for in vitro selection of antibodies [7]. With this approach, it is possible to select mAbs of virtually any specificity in vitro, thereby providing mAbs not only for therapeutic use in humans but also for use in research and clinical diagnostics [8].

In this study, a phage display library of human singlechain variable fragment $(\mathrm{scFv})$ antibodies was constructed from the B cells of healthy volunteers boosted by the VACV vaccine. Human scFvs against VACV were then isolated, and their neutralizing efficacy was demonstrated following conversion to human immunoglobulin G1 (IgG1).

\section{Materials and Methods}

\section{Blood collection and RNA isolation}

Five healthy volunteers were vaccinated with a smallpox vaccine (Lancy-Vaxina vaccine; Berna Biotech, Switzerland) once. After 7 days of vaccination, $20 \mathrm{ml}$ of blood was collected from each individual using a vacutainer tube (Becton Dickinson, USA) containing sodium heparin. Vaccination and blood collection were approved by the Institutional Review Board of Seoul National University Hospital (IRB No. H-0606-015-175).

Isolation of lymphocytes from the blood, preparation of total RNA, and cDNA synthesis were performed as previously described [9].

Construction of an antibody library and screening of antiVACV scFvs

DNA was amplified using Ready-To-Go RT-PCR Beads (GE Healthcare Bio-Sciences, USA) according to the manufacturer's

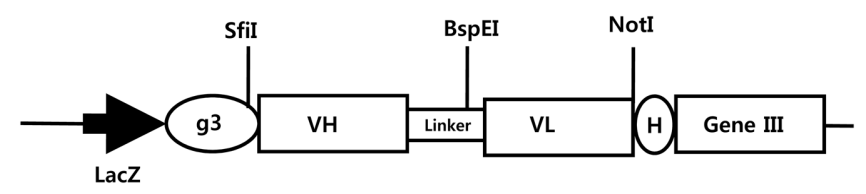

Fig. 1. Arrangement of genes in the $\mathrm{pKS} 4 \mathrm{H}$ vector.

LacZ denotes the lacZ promoter, and $\mathrm{g} 3$ denotes the leader peptide of gene III of filamentous phage M13. VH and VL denote variable regions of the immunoglobulin $\mathrm{H}$ and $\mathrm{L}$ chains, respectively. $\mathrm{H}$ denotes the $6 \times$ His tag. Gene III denotes the gene that encodes a minor coat protein of filamentous phage M13. Restriction enzyme sites used for cloning scFv are shown.

instructions. Primers were designed to fit into the N- and Cterminal regions of each variable heavy chain $(\mathrm{VH})$, variable kappa chain $(\mathrm{V} \kappa)$, and variable lambda chain $(\mathrm{V} \lambda)$ region (Table 1). VHs were amplified with a mixture of primers JH1, JH2 and $\mathrm{JH} 3$ and with primers $\mathrm{VH} 1, \mathrm{VH} 3$, and $\mathrm{VH} 4$, respectively, followed by another round of polymerase chain reaction (PCR) with primers VH Ext and LINK2. Vкs were amplified with a

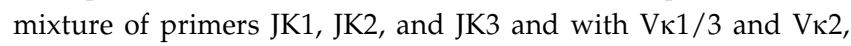
followed by another round of PCR with primers JK Ext and LINK1. V $\lambda$ s were amplified with a mixture of primers $J \lambda 1$ and $J \lambda 2$ and a mixture of primers $V \lambda 1$ and $V \lambda 3$, followed by another round of PCR with primers J $\lambda$ Ext and LINK1. Purification of PCR products, cloning into pKS4H (Fig. 1), and preparation of a phagedisplayed antibody library were performed as previously described [9].

ScFvs that bind to VACV were screened by panning in immunotubes (Maxisorp; NUNC A/S, Denmark) as previously described [10]. Tubes were prepared by coating with $1 \mathrm{ml}$ of Vaccinia (Lister strain) purified virus (Advanced Biotechnologies, Inc., USA) diluted up to $2.5 \mu \mathrm{g} / \mathrm{ml}$ in phosphate-buffered saline (PBS).

Expression of scFv in Escherichia coli, and ELISA to measure scFv binding to VACV

Expression of $\mathrm{scFv}$ in colonies obtained from the fourth round of panning and ELISA for measuring the binding of $\mathrm{scFv}$ to VACV were performed as previously described [11]. VACV was diluted to $2.5 \mu \mathrm{g} / \mathrm{ml}$ in PBS and immobilized in 96-well plates (Nunc Immuno Module, Maxisorp; NUNC A/S). ScFvs bound to VACV were detected using the 6x-His Epitope Tag Antibody, HRP conjugate (Thermo Fisher Scientific, USA) and 3,3',5,5'tetramethylbenzidine (TMB) solution (KPL, USA) as a substrate. The sequencing of colonies displaying binding to VACV in ELISA was performed by Genotech (Korea) using primer P035 (Table 1). Two scFv clones, SC34 and SC212, were screened based on their binding to VACV and by sequence analysis for the next step.

Establishment of a cell line producing human anti-VACV IgG For construction of the VH-expression vector, the signal 
Table 1. Oligonucleotide primers used for cloning and sequencing of human immunoglobulin genes.

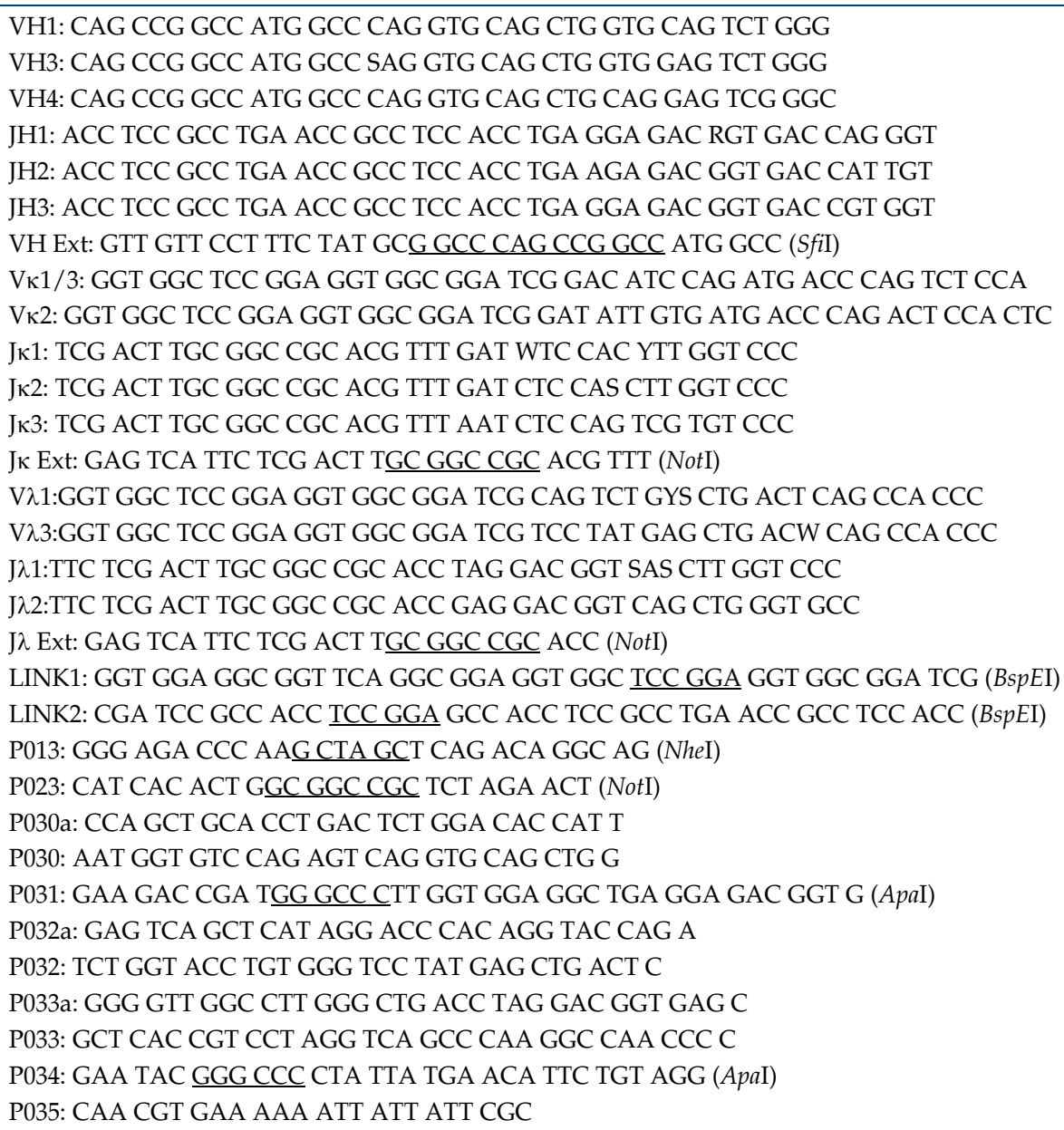

${ }^{a}$ The primer sequences are shown in the $5^{\prime}-3^{\prime}$ direction. S denotes G or C. W denotes A or T. Y denotes C or T. The restriction enzymes used for cloning are shown in parentheses, and their recognition sites are underlined.

sequence (Sig) of pRC13 (Fig. 2) [12] was amplified by PCR using primers P023 and P030a (all primers are listed in Table 1), and the VH sequence was amplified by PCR using primers P030 and P031 on the scFvs described above (SC212 and SC34). The two

A

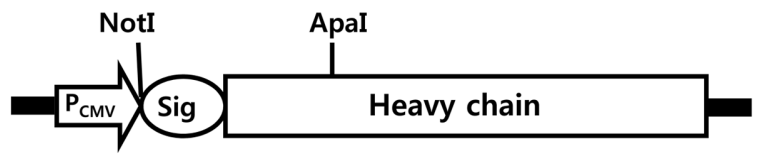

B

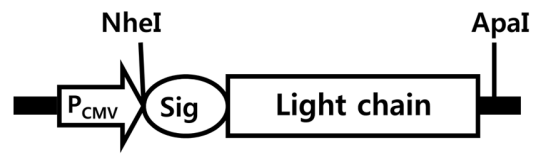

Fig. 2. Arrangement of genes in expression vectors. pRC13 for heavy chain (A) and pKC12 for light chain (B). Restriction enzyme sites used for cloning are shown. $P_{\mathrm{CMV}}$, cytomegalovirus promoter; Sig, signal peptide of human immunoglobulin. amplified fragments (Sig and VH) were then fused by PCR using primers P023 and P031, and this Sig-VH PCR fragment was inserted into pRC13 following digestion with NotI and ApaI.

For construction of the variable light chain (VL) expression vector, Sig of pKC12 [12] was amplified by PCR using primers P013 and P032a (Fig. 2) [12], and the VL was amplified by PCR using primers P032 and P033a on SC212 and SC34. The two fragments (Sig and VL) were then fused by PCR using primers P013 and P033a. The lambda constant region (CL) was amplified by PCR using primers $\mathrm{P} 033$ and P034 from the CDNA of human B lymphocytes prepared for library construction. The CL PCR fragment was fused to the Sig-VL PCR fragment by PCR using primers P013 and P034, and this Sig-VL-CL PCR fragment was inserted into $\mathrm{pKC} 12$ following digestion with NheI and ApaI.

The CHO DG44 cell line expressing human anti-VACV IgG was established as previously described [12]. The established cell lines VC34 and VC212 were adapted to serum-free medium P1 (GC Pharma, Korea), and antibodies were produced in a 
disposable-wave bioreactor (GE Healthcare). The mAbs of VC34 and VC212 were purified from the culture media using protein A agarose beads (GE Healthcare), followed by ion-exchange chromatography and verification of antibody purity by SDSPAGE.

\section{Determination of affinities of selected clones}

The affinities of anti-VACV mAbs (VC34 and VC212) were determined by competition ELISA, as described previously [13], using VACV-coated plates and free VACV. The binding affinity $\left(\mathrm{IC}_{50}\right)$ was defined as the VACV concentration that provides $50 \%$ inhibition of anti-VACV mAb binding to immobilized VACV as compared with maximum binding, i.e., ELISA readings performed in the absence of competitive VACV.

\section{Determination of VACV neutralization by anti-VACV $\mathrm{mAb}$}

VACV neutralization by anti-VACV mAb was determined by a plaque-reduction neutralization test (PRNT) [14]. For PRNT, $1 \times 10^{6}$ Vero E6 cells (Korea Centers for Disease Control and Prevention (KCDC), Korea) were seeded into 6-well plates and incubated overnight. The $\mathrm{VACV}_{\mathrm{WR}}$ strain (KCDC) was diluted to $2 \mathrm{pfu} / \mu \mathrm{l}$ in Eagle's minimum essential medium (EMEM; Lonza, USA) supplemented with $1 \%$ fetal bovine serum (FBS). AntiVACV mAbs were 2-fold serially diluted (up to 1:1024) with EMEM containing $1 \%$ FBS. The starting concentrations of $\mathrm{mAb} \mathrm{VC} 34$ and $\mathrm{mAb}$ VC212 were $2.9 \mathrm{mg} / \mathrm{ml}$ and $2.4 \mathrm{mg} / \mathrm{ml}$, respectively. Equal volumes $(120 \mu \mathrm{l})$ of diluted mAbs and VACV were then mixed and incubated at $37^{\circ} \mathrm{C}$ for $18 \mathrm{~h}$.

The 6-well plates were washed twice with PBS, and $200 \mu 1$ of the virus $/ \mathrm{mAb}$ mixture was added and incubated at $37^{\circ} \mathrm{C}$ in $5 \% \mathrm{CO}_{2}$ for $90 \mathrm{~min}$. Medium was aspirated, and cells were overlaid with $2 \mathrm{ml}$ of overlay medium [1:1 mixture of $2 \times$ MEM containing $2 \%$ FBS and $1 \%$ agarose (SeaPlaque agarose; Lonza)] and incubated for 2 days. After the medium was aspirated, cells were fixed with $10 \%$ formaldehyde for $1 \mathrm{~h}$, stained with crystal violet, and washed once with PBS, and plaques were then counted. The $\mathrm{PRNT}_{50}$ was defined as the $\mathrm{mAb}$ concentration that caused a $50 \%$ reduction in the number of VACV plaques as compared with the number of $\mathrm{VACV}$ plaques obtained from culture media.

\section{Results}

\section{Amplification of DNA and library construction}

Total RNA was prepared from the peripheral blood of smallpox-vaccine-immunized healthy volunteers. A group of primers targeting the $\mathrm{N}$ - and C-termini of the $\mathrm{VH}$ and

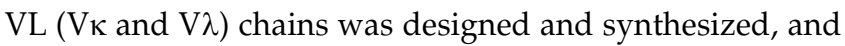
the subsequent PCR products appeared at the expected size of $\sim 350$ bp by agarose gel electrophoresis (Fig. 3). The fragments were inserted into the phagemid vector $\mathrm{pKS} 4 \mathrm{H}$ (Fig. 1), in which the lacZ promoter drives expression, and the g3 leader sequence guides secretion of scFvs. Gene III,

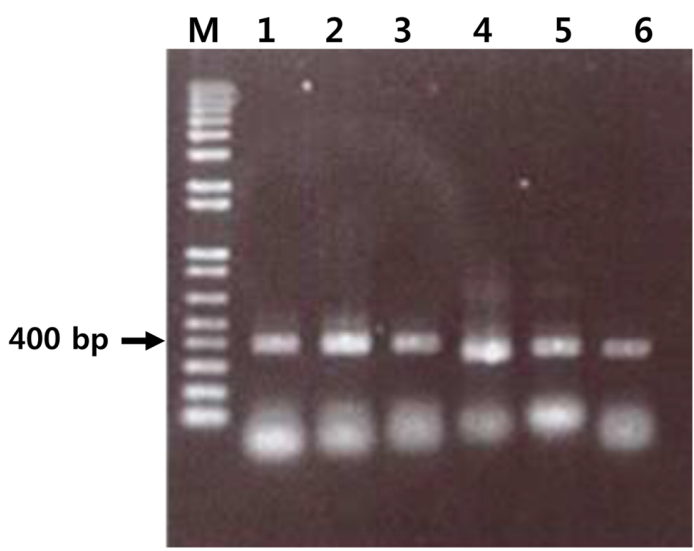

Fig. 3. Analysis of amplified PCR products.

Agarose gel (1.2\%) analysis of PCR fragments of $\mathrm{VH}, \mathrm{V} \kappa$, and $\mathrm{V} \lambda$ chains. M: DNA ladder (Life Technologies, Carlsbad, CA, USA); lane 1: VH1; lane 2: VH3; lane 3: VH4; lane 4: Vк1/3; lane 5: Vк2; lane 6: $\mathrm{V} \lambda 1$ and $\mathrm{V} \lambda 3$.

which encodes the minor coat protein of filamentous phage $\mathrm{M} 13$, is positioned at the C-terminus of $\mathrm{scFv}$, thereby allowing $\mathrm{ScFv}$ to be displayed on the surface of the phage particles.

The library was constructed by inserting $\mathrm{VH}$ first, followed by $\mathrm{VL}$, into the phagemid $\mathrm{pKS} 4 \mathrm{H}$, resulting in a library size of $\sim 1.0 \times 10^{9}$. Library diversity was confirmed by DNA sequencing of individual colonies from the library (data not shown).

\section{Selection of scFvs that bind to VACV}

For selection of anti-VACV scFvs, four rounds of panning were performed in the VACV-immobilized immunotubes, and $\mathrm{scFv}$ expression was induced in the colonies from the fourth round of panning. ScFvs were expressed in E.coli XL1 Blue cells and secreted into the culture medium, and ELISA was performed on VACV-immobilized plates to confirm the binding of the secreted scFvs to VACV. Two clones, SC34 and SC212, were selected, and their sequences analyzed (Fig. 4), revealing that both scFvs possessed V $\lambda$.

\section{Conversion of scFv to human IgG1 and estimation of binding affinity}

The VH and VL of SC34 and SC212 obtained from library screening were converted to human $\operatorname{IgG}_{1}$ (mAb VC34 and $\mathrm{mAb}$ VC212) via insertion into the mammalian expression vectors pRC13 (VH) and pKC12 (VL), respectively. Because the original form of $\mathrm{pKC} 12$ contained the constant region of the kappa chain, the lambda constant region was cloned from the cDNA of B lymphocytes. 

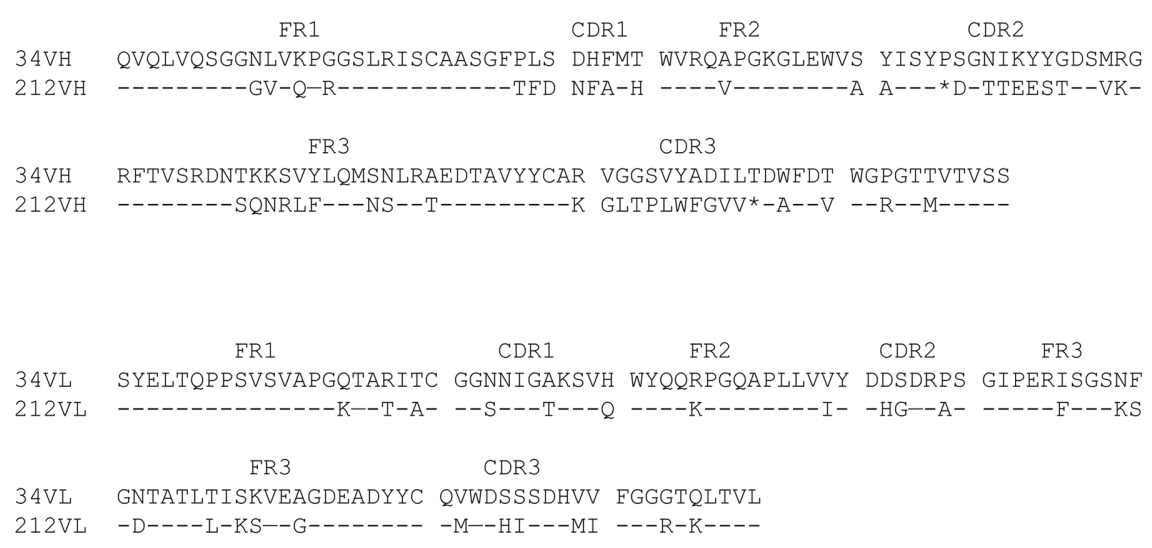

Fig. 4. Amino acid sequences of VH and VL of human anti-VACV mAbs SC34 and SC212.

Complementarity-determining regions (CDR1, CDR2, and CDR3) and framework regions (FR1, FR2, and FR3) are indicated above the sequence alignment. Dashes (-) indicate an identical residue. Asterisks $\left(^{*}\right)$ indicate deletion of amino acids.

CHO DG-44 cells were co-transfected with the $\mathrm{H}$ and $\mathrm{L}$ vectors, selected against geneticin, and growth-adapted up to $1 \mu \mathrm{M}$ methotrexate. Cells were then adapted to serumfree suspension culture, and the antibodies were purified using protein A agarose and ion-exchange chromatography.

The binding affinities of VC34 and VC212 were compared by competition ELISA, yielding $\mathrm{IC}_{50}$ values estimated at $2 \mu \mathrm{g} / \mathrm{ml}(13.33 \mathrm{nM})$ and $22 \mu \mathrm{g} / \mathrm{ml}(146.67 \mathrm{nM})$, respectively (Fig. 5). VC34 exhibited 11-fold higher affinity to VACV as compared with VC212.

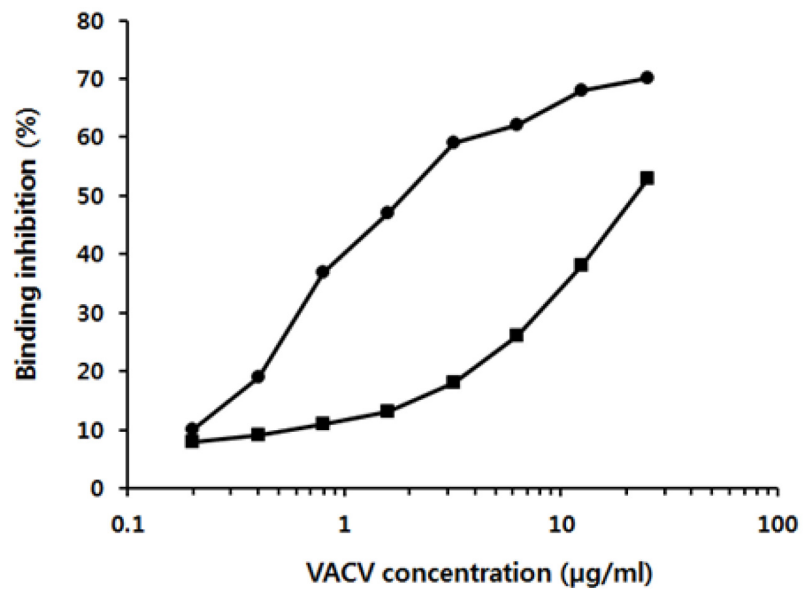

Fig. 5. Competition ELISA for measuring relative affinities of anti-VACV mAbs.

Inhibition of anti-VACV $\mathrm{mAb}$ binding to VACV-coated plates by free VACV was analyzed using different concentrations of free VACV. Each symbol denotes a clone of VC34 (-) and VC212 Competition ELISA was performed only once due to a lack of VACV availability.

\section{VACV neutralization by anti-VACV $\mathrm{mA}$}

VACV neutralization by anti-VACV mAbs was determined by PRNT. The $\mathrm{PRNT}_{50}$ was defined as the $\mathrm{mAb}$ concentration that reduced the number of VACV plaques by $50 \%$ as compared with the number of VACV plaques using the culture medium for $\mathrm{CHO}$ cells. The $\mathrm{PRNT}_{50}$ of VC212 was estimated at $\sim 0.16 \mathrm{mg} / \mathrm{ml}(\sim 1.07 \mu \mathrm{M})$ (Fig. 6), whereas VC34 did not exhibit plaque count reduction, but showed an 11-fold higher affinity to VACV relative to VC212.

\section{Discussion}

There have been several attempts to screen recombinant VIGs from the phage-display antibody libraries of different genetic sources and antibody formats, such as phagedisplay chimpanzee Fab libraries $[15,16]$, the phagedisplay human Fab library [17], and the phage-display human scFv library [18]. In addition, human mAbs against VACV antigens were screened using KM mice carrying the human chromosome fragments containing the entire human immunoglobulin heavy-chain locus and the YAC transgene for $50 \%$ of the human immunoglobulin kappa light-chain locus, and used to select human mAbs against VACV antigens [19].

Chimpanzee Fabs against the VACV B5 envelope protein were isolated from a phage display Fab library derived from the bone marrow of chimpanzees vaccinated with VACV and then converted to mAbs with human IgG1 heavy-chain constant regions (8AH8AL and 8AH7AL with $K_{d}$ values of 0.2 and $0.7 \mathrm{nM}$ respectively) [15]. The authors demonstrated that mAbs against VACV B5 envelope protein alone protected mice sufficiently from lethal 
A

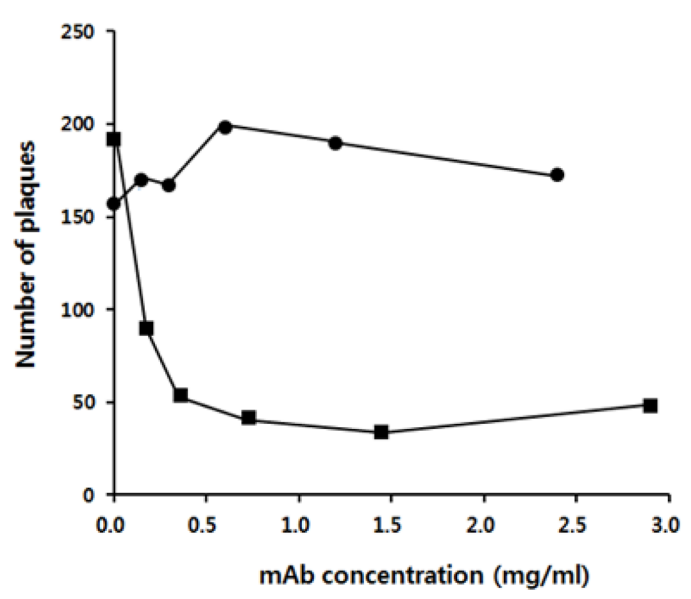

B
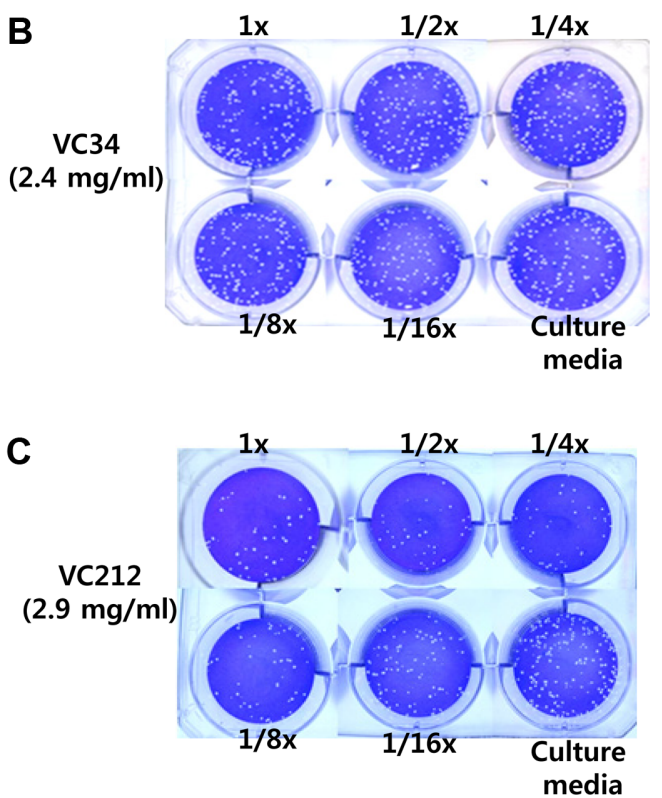

Fig. 6. Neutralization of VACV by anti-VACV mAb as determined by PRNT.

(A) Plot of the number of plaques versus mAb concentration. Each symbol denotes VC34 (-) and VC212 ( $\mathbf{\square})$. PRNT for VC34 (B) and VC212 (C), with initial concentrations of $2.4 \mathrm{mg} / \mathrm{ml}$ and $2.9 \mathrm{mg} / \mathrm{ml}$, respectively, both of which were diluted 16-fold. For PRNT, Vero E6 cells and the $\mathrm{VACV}_{\text {WR }}$ strain were employed. The PRNT assay was performed only once due to a lack of VACV availability.

challenge with virulent VACV and mice administered 2 days after infection [15]. The efficacy of 8AH8AL and 8AH7AL could not be compared to the efficacy of VC212 due to the absence of PRNT of these mAbs. Human VIG was found to be inferior to chimpanzee-human mAbs in animal studies because of the low concentration of protective antibodies in VIG; $5 \mathrm{mg}$ of VIG contains less than $10 \mu \mathrm{g}$ of $\mathrm{mAb}$ to the $\mathrm{B} 5$ envelope protein [15].

Chimpanzee Fabs against the VACV A33 envelope protein were isolated from the same chimpanzee Fab phage library described above, and then converted to $\mathrm{mAbs}$ with human IgG1 heavy-chain constant regions $(6 \mathrm{C}, 12 \mathrm{C}$, and $12 \mathrm{~F}$ with $K_{d}$ values of $0.14-20 \mathrm{nM}$ ) [16]. Two chimpanzeehuman $\mathrm{mAbs}(6 \mathrm{C}$ and $12 \mathrm{~F})$ showed similar protective efficacy, although $6 \mathrm{C}$ exhibited $~ 140$-fold lower affinity for VACV A33 envelop protein than 12F [16]. It was also shown that similar protection was achieved with the mAbs against the VACV A33 envelope protein compared to that with the mAbs against the VACV B5 envelope protein [16].

Human Fabs against VACV were isolated from a phagedisplay library constructed with lymphocytes of vaccinated donors [17]. The authors collected B cells from vaccinated donors as in the current study, and then constructed the Fab phage library as opposed to the scFv phage library in the current study. They selected 6 Fab clones representing neutralization of VACV by PRNT. However, they did not show the PRNT with IgG mAbs.

Human scFvs capable of neutralizing VACV were selected from a human scFv phage library by panning against VACV [18]. Construction of the scFv phage library and panning against VACV were very similar to those in the current study; however the authors tested the neutralization of VACV with phage antibodies, which was different from the IgGs that we measured.

Human mAbs against H3 and B5 VACV antigens were produced using KM mice [19]. The authors found that combination therapy with two fully human mAbs against $\mathrm{H} 3$ and B5 provided significantly better protection against VACV infection in a small animal model of progressive vaccinia (SCID mice infected with $\mathrm{VACV}_{\mathrm{NYCBOH}}$ vaccine strain) compared to the use of single human mAbs or VIG. The $\mathrm{PRNT}_{50}$ of human anti-H3 mAb hV26 was estimated to be $\sim 100 \mathrm{ng} / \mathrm{ml}(\sim 0.67 \mathrm{nM})$ for neutralization of $\mathrm{VACV}_{\mathrm{WR}}$ according to the standard overnight neutralization assay. The PRNT ${ }_{50}$ of VC212 in this investigation against the same $\mathrm{VACV}_{\text {WR }}$ strain was $\sim 0.16 \mathrm{mg} / \mathrm{ml}(\sim 1.07 \mu \mathrm{M})$, indicating much lower neutralization efficiency of VC212 compared to that of hV26. In addition, the authors showed that in vivo efficacy of $100 \mu \mathrm{g}$ of human anti-H3 mAb hV26 was similar to that of $1.25 \mathrm{mg}$ VIG. SCID mice treated with a 
single dose of $100 \mu \mathrm{g}$ human anti-H3 mAb hV26 and $1.25 \mathrm{mg}$ VIG at day-1 followed by infection with $1 \times 10^{4} \mathrm{PFU}$ $\mathrm{VACV}_{\mathrm{WR}}, \mathrm{mAb}$ hV26 and VIG exhibited similar in vivo protection, as revealed by body weight, \% survival, and clinical score [19].

This study solved the disadvantages of human plasmaderived VIG production by isolating human mAbs capable of neutralizing VACV from a phage-display scFv library prepared from B cells of VACV-vaccinated volunteers. As a result, a human anti-VACV mAb VC212 having a $\mathrm{PRNT}_{50}$ of $\sim 0.16 \mathrm{mg} / \mathrm{ml}(\sim 1.07 \mu \mathrm{M})$ was selected. This VC212 provides the basis for further development of VACV-neutralizing human mAbs for overcoming vaccine complications and as agents for prevention of disease after exposure to smallpox.

\section{Acknowledgments}

This study was supported by a research grant from the Korea Centers for Disease Control \& Prevention (KCDC) (grant number: 4800-4840-300).

\section{Conflict of Interest}

The authors have no financial conflicts of interest to declare.

\section{References}

1. Walsh SR, Dolin R. 2011. Vaccinia viruses: vaccines against smallpox and vectors against infectious diseases and tumors. Expert. Rev. Vaccines 10: 1221-1240.

2. Moore ZS, Seward JF, Lane JM. 2006. Smallpox. Lancet 367: 425-435.

3. Lustig S, Maik-Rachline G, Paran N, Melamed S, Israely T, Erez $\mathrm{N}$, et al. 2009. Effective post-exposure protection against lethal orthopoxviruses infection by vaccinia immune globulin involves induction of adaptive immune response. Vaccine 27: 1691-1699

4. Wittek R. 2006. Vaccinia immune globulin: current policies, preparedness, and product safety and efficacy. Int. J. Infect. Dis. 10: 193-201.

5. Prescribing Information of CNJ-016, Vaccinia Immune Globulin Intravenous (Human), sterile solution. Emergent BioSolutions (https:/ /www.emergentbiosolutions. com/sites/ default/files/inline-files/LBL040011.pdf).

6. Schmaljohn C, Cui Y, Kerby S, Pennock D, Spik K. 1999. Production and characterization of human monoclonal antibody Fab fragments to vaccinia virus from a phagedisplay combinatorial library. Virology 258: 189-200.
7. Frenzel A, Schirrmann T, Hust M. 2016. Phage-displayderived human antibodies in clinical development and therapy. MAbs 8: 1177-1194.

8. Hammers CM, Stanley J Jr. 2014. Antibody phage display: technique and applications. J. Invest. Dermatol. 134: 1-5.

9. Kim SH, Park SY. 2002. Selection and characterization of human antibodies against hepatitis B virus surface antigen (HBsAg) by phage-display. Hybrid. Hybridomics 21: 385-392.

10. Kim SH, Titlow CC, Margolies MN. 2000. An approach for preventing recombination-deletion of the 40-50 anti-digoxin antibody $\mathrm{V}(\mathrm{H})$ gene from the phage display vector $\mathrm{pComb3}$. Gene 241: 19-25.

11. Kim SH, Song SH, Kim YJ, Park SY. 2001. Expression and characterization of a recombinant Fab fragment derived from an anti-human alpha-fetoprotein monoclonal antibody. Mol. Cells. 11: 158-163.

12. Shin YW, Ryoo KH, Hong KW, Chang KH, Choi JS, So M, et al. 2007. Human monoclonal antibody against Hepatitis B virus surface antigen (HBsAg). Antiviral Res. 75: 113-120.

13. Kim SH, Chun JH, Park SY. 2001. Characterization of monoclonal antibodies against carcinoembryonic antigen (CEA) and expression in E. coli. Hybridoma 20: 265-272.

14. Borges MB, Kato SE, Damaso CR, Moussatché N, da Silva Freire M, Lambert Passos SR, et al. 2008. Accuracy and repeatability of a micro plaque reduction neutralization test for vaccinia antibodies. Biologicals 36: 105-110.

15. Chen Z, Earl P, Americo J, Damon I, Smith SK, Zhou YH, et al. 2006. Chimpanzee/human mAbs to vaccinia virus B5 protein neutralize vaccinia and smallpox viruses and protect mice against vaccinia virus. Proc. Natl. Acad. Sci. USA 103: 1882-1887.

16. Chen Z, Earl P, Americo J, Damon I, Smith SK, Yu F, et al. 2007. Characterization of chimpanzee/human monoclonal antibodies to vaccinia virus A33 glycoprotein and its variola virus homolog in vitro and in a vaccinia virus mouse protection model. J. Virol. 81: 8989-8995.

17. Schmaljohn C, Cui Y, Kerby S, Pennock D, Spik K. 1999. Production and characterization of human monoclonal antibody Fab fragments to vaccinia virus from a phagedisplay combinatorial library. Virology 258: 189-200.

18. Tikunova NV, Morozova VV, Batanova TA, Belanov EF, Bormotov NI, Ilyichev AA. 2001. Phage antibodies from combinatorial library neutralize vaccinia virus. Hum. Antibodies 10: 95-99.

19. McCausland MM, Benhnia MR, Crickard L, Laudenslager J, Granger SW, Tahara T, et al. 2010. Combination therapy of vaccinia virus infection with human anti-H3 and anti-B5 monoclonal antibodies in a small animal model. Antivir. Ther. 15: 661-675. 